\title{
Coarsening dynamics of three-dimensional levitated foams: From wet to dry
}

\author{
Isert, $\mathrm{N}$; Maret, G ; Aegerter, C M
}

\begin{abstract}
We study diamagnetically levitated foams with widely different liquid fractions. Due to the levitation, drainage is effectively suppressed and the dynamics is driven by the coarsening of the foam bubbles. For dry foams, the bubble size is found to increases as the square root of foam age, as expected from a generalized von Neumann law. At higher liquid content the behavior changes to that of Ostwald ripening where the bubbles grow with the $1 / 3$ power of the age. Using Diffusing Wave Spectroscopy we study the local dynamics in the different regimes and find diffusive behavior for dry foams and kinetic behavior for wet foams.
\end{abstract}

DOI: https://doi.org/10.1140/epje/i2013-13116-x

Posted at the Zurich Open Repository and Archive, University of Zurich ZORA URL: https://doi.org/10.5167/uzh-90125

Journal Article

Published Version

Originally published at:

Isert, N; Maret, G; Aegerter, C M (2013). Coarsening dynamics of three-dimensional levitated foams: From wet to dry. European Physical Journal. E, Soft matter, 36(10):116.

DOI: https://doi.org/10.1140/epje/i2013-13116-x 


\title{
Coarsening dynamics of three-dimensional levitated foams: From wet to dry
}

\author{
N. Isert ${ }^{1}$, G. Maret ${ }^{1}$, and C.M. Aegerter ${ }^{2, a}$ \\ 1 Fachbereich Physik, Universität Konstanz, Universitätsstrasse 10, 78457 Konstanz, Germany \\ 2 Physics Institute, University of Zurich, Winterthurerstr. 190, 8057 Zurich, Switzerland
}

Received 20 February 2013 and Received in final form 17 May 2013

Published online: 17 October 2013 - (C) EDP Sciences / Società Italiana di Fisica / Springer-Verlag 2013

\begin{abstract}
We study diamagnetically levitated foams with widely different liquid fractions. Due to the levitation, drainage is effectively suppressed and the dynamics is driven by the coarsening of the foam bubbles. For dry foams, the bubble size is found to increases as the square root of foam age, as expected from a generalized von Neumann law. At higher liquid content the behavior changes to that of Ostwald ripening where the bubbles grow with the $1 / 3$ power of the age. Using Diffusing Wave Spectroscopy we study the local dynamics in the different regimes and find diffusive behavior for dry foams and kinetic behavior for wet foams.
\end{abstract}

Foams are models of soft matter consisting of gas bubbles enclosed in a liquid which have solid properties due to surface tension of the bubbles and their inside pressure [1]. Depending on the amount of liquid in the foam, bubbles can either be in contact or separated. In the former most common case of "dry" or "almost dry" foams, bubbles are substantially deformed and the thin flat liquid films in the contact area between them provide substantial mechanical stability to the foam [1]. "Wet" foams are less stable because the rather homogeneous mixture of separated bubbles is rapidly destroyed by flows due to buoyancy pushing bubbles upwards [1]. In "wet" and "almost dry" foams the dynamics is driven by the drainage of liquid between the bubbles due to gravity [2]. However, additional dynamics occurs even without drainage because of gas exchange between bubbles $[3,4]$ due to the Laplace pressure $\Delta p=2 \sigma / r$ with $r$ being the bubble's radius of curvature and $\sigma$ the liquid-gas surface tension [5]. As a consequence, the average bubble size increases with time because of the lower gas pressure in the larger bubbles. This process, known as coarsening, has been described by von Neumann [6] and has been experimentally observed in two-dimensional foams [3] as well as for very dry foams [7, 8]. It has also been shown that drainage and coarsening can interact $[9,10]$.

Here we study three-dimensional foams which are levitated by a strong magnetic field gradient [11,12]. Due to the diamagnetism of water, it is possible to effectively suppress the buoyancy of the gas bubbles and thus stabilize the foam against drainage even at high liquid content. With this simple trick it becomes possible to study the

\footnotetext{
a e-mail: aegerter@physik.uzh.ch
}

coarsening dynamics in 3D foams without chemical stabilizers for dry as well as wet foams over many hours in laboratory. Without levitation such foams would decay within minutes on Earth [13] which is why major efforts are under way to investigate them at conditions of steady microgravity at the International Space Station [14].

In dry foams the exchange of gas between bubbles takes place directly through the thin liquid films separating the bubbles [15]. Because the rate of exchange is governed by the Laplace pressure $\Delta p$, the growth rate of a bubble, i.e. the current density of gas exchange, is proportional to $\Delta p$, i.e. to the inverse of its radius. This implies $j=\frac{\mathrm{d} V}{A \mathrm{~d} t} \propto \mathrm{d} r / \mathrm{d} t \propto 1 / r$. Here, $A$ is the contact area of a bubble which is of order $r^{2}$ and $V$ its volume. Given this dynamics, one obtains after integration that the average size of bubbles will increase with time as $\langle r\rangle \propto t^{1 / 2}$. This can also be derived more rigorously, as for instance done in [16], where strictly speaking the pressure difference between two different bubbles is considered. This implies that one has to consider a state of the bubbles where the distribution is random giving rise to variations in the pressure difference, such that coarsening can take place. For the argument to hold, the distribution of bubbles during coarsening has to be statistically self-similar, something which may only appear after a long time depending on the initial state of the foam after production.

When the bubbles are no longer in contact, it can be surmised that the mechanism of gas exchange between bubbles will have to change. In fact, the exchange of gas will now have to be achieved via diffusion in the liquid and the difference of the gas pressure in the bubbles to the saturation pressure in the liquid. Here, the diffusive current density $j \propto \mathrm{d} r / \mathrm{d} t$ will be determined by the gradient 
of the concentration, i.e. the gradient in pressure difference. This means that we obtain $j \propto \mathrm{d} r / \mathrm{d} t \propto \mathrm{d} p / \mathrm{d} r$. Again using the fact that the pressure inside the bubbles is given by Laplace's law, we obtain $\mathrm{d} r / \mathrm{d} t \propto 1 / r^{2}$ and hence a growth of the form $\langle r\rangle \propto t^{1 / 3}$. Again, this can be derived studying the detailed dynamics [17-21]. This qualitatively different type of coarsening is also known as Ostwald ripening and is for instance observed in the dynamics of the growth of inclusions in solids [22]. Since the nature of the dynamics changes when the bubbles are no longer in contact, the boundary between the two regimes is expected to be at a liquid fraction of $\sim 30 \%$, which corresponds to that of the inverse structure of closely packed spheres (26\% for ordered structures, $36 \%$ for disordered structures). This transition has also been obtained in simulations of two-dimensional foams considering different liquid fractions [23]. Since levitated foams can be created with a varying amount of liquid and relative stability to drainage, these predictions can thus be tested experimentally with our setup.

In order to assess the growth of the foam bubbles with age inside the three-dimensional foam we use multiply scattered light transmitted through the foam [24]. The transport mean free path of light in the turbid medium of the foam has been found to be proportional to the bubble size [26-28]. This holds both for dry foams, where scattering mainly takes place at the thin liquid films between bubbles as well as for liquid foams, where the scattering centers are the bubbles themselves. For comparison we use direct visualization by video microscopy of bubbles near the sample surface. In addition, the transmitted diffuse light can be used to obtain information on the averaged local dynamics of the foam via Diffusing Wave Spectroscopy (DWS) [29]. Here, the auto-correlation function of the multiply scattered light field directly provides the time dependence of the optical phase shift incurred by the local motions of the scattering particles. Thus it is possible to determine whether the dynamics of the scatterers is diffusive or ballistic and from the respective time scales [30], the size of the dynamic particles can be obtained.

The foams used in the experiments consist of water, sodium dodecyl sulfate (SDS) as a surfactant and $\mathrm{N}_{2}$ gas with a surfactant concentration of $8 \%$ by weight. The water-SDS mixture and the gas are put in two separate syringes, which are connected through a thin tube [31]. The water-SDS mixture is then transferred to the gas-filled syringe and the resulting mixture is transferred through the thin tube several times in order to achieve turbulent mixing. In this way an irregular foam with a given mean pressure in the bubbles determined by the pressure on the syringes is obtained. This irregular initial state ensures a quick approach to a statistically self-similar state and thus the asymptotic coarsening regime. The liquid content in the foam is determined by the ratio of the volume of waterSDS mixture and that of gas initially present within the two syringes [31]. Due to the compression of the gas in the foam bubbles, the effective liquid fraction of the foam will be somewhat higher than the initial composition given by the content in the two syringes. An initial liquid fraction of $25 \%$ corresponds roughly to an effective liquid fraction
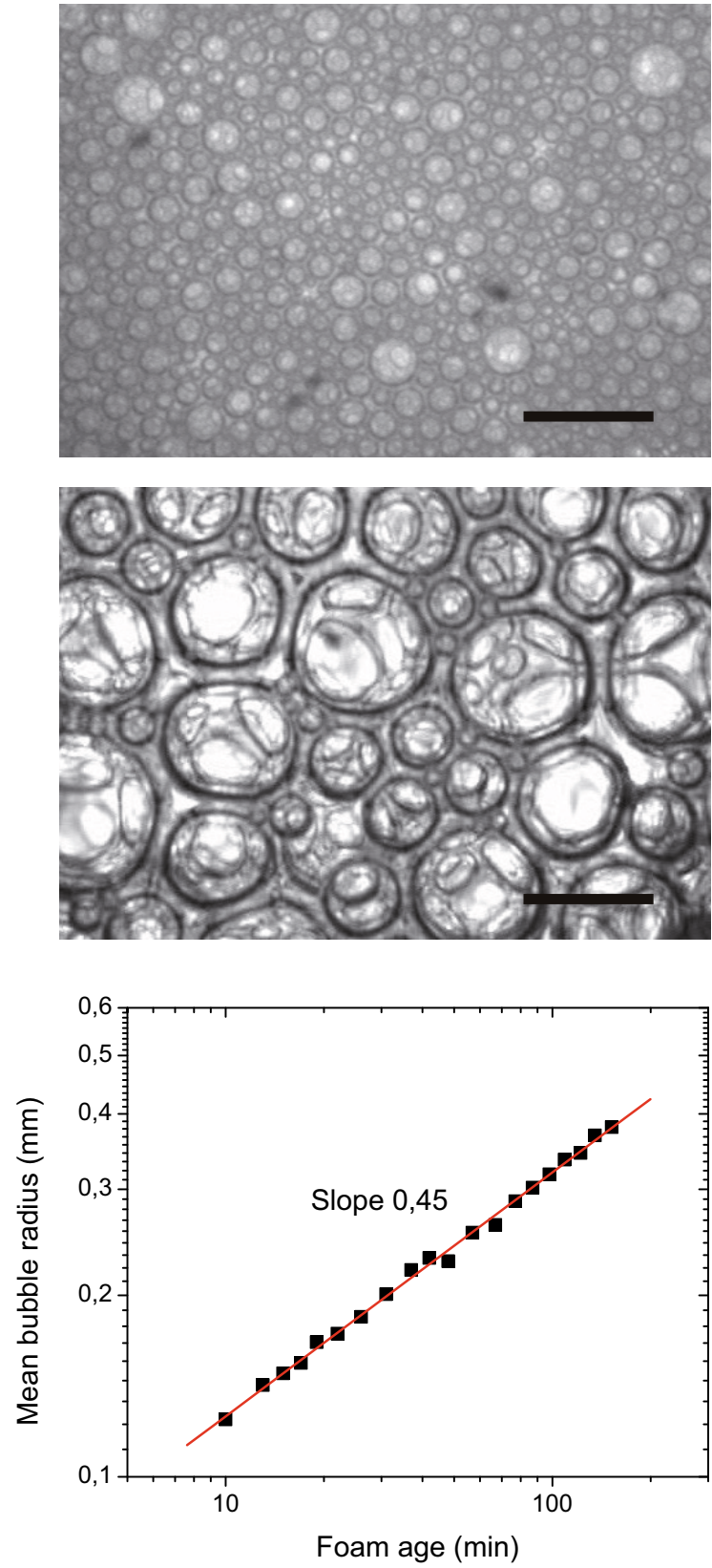

Fig. 1. Image of the surface of the foam chamber at different times during the ageing of the foam with an initial liquid fraction of $20 \%$. This shows the absolute size and polydispersity of the foam. The scale bar corresponds to $1 \mathrm{~mm}$. Shown are images $10 \mathrm{~min}$ (top) and $152 \mathrm{~min}$ after creation (middle). The average radius of the bubbles as a function of time is shown in the bottom figure, showing a growth law $\langle r\rangle \propto t^{\beta}$ with an exponent $\beta=0.45(5)$.

of $30 \%$. This can be estimated from the pressure exerted by the foam in the syringes. The polydisperse foam with an average bubble radius of $r_{0} \simeq 100 \mu \mathrm{m}$ (see fig. 1a) thus created is then transferred to a sample cell of diameter of $1.7 \mathrm{~cm}$ and height of $1.2 \mathrm{~cm}$, which is placed inside the room temperature bore of a superconducting cryomagnet 


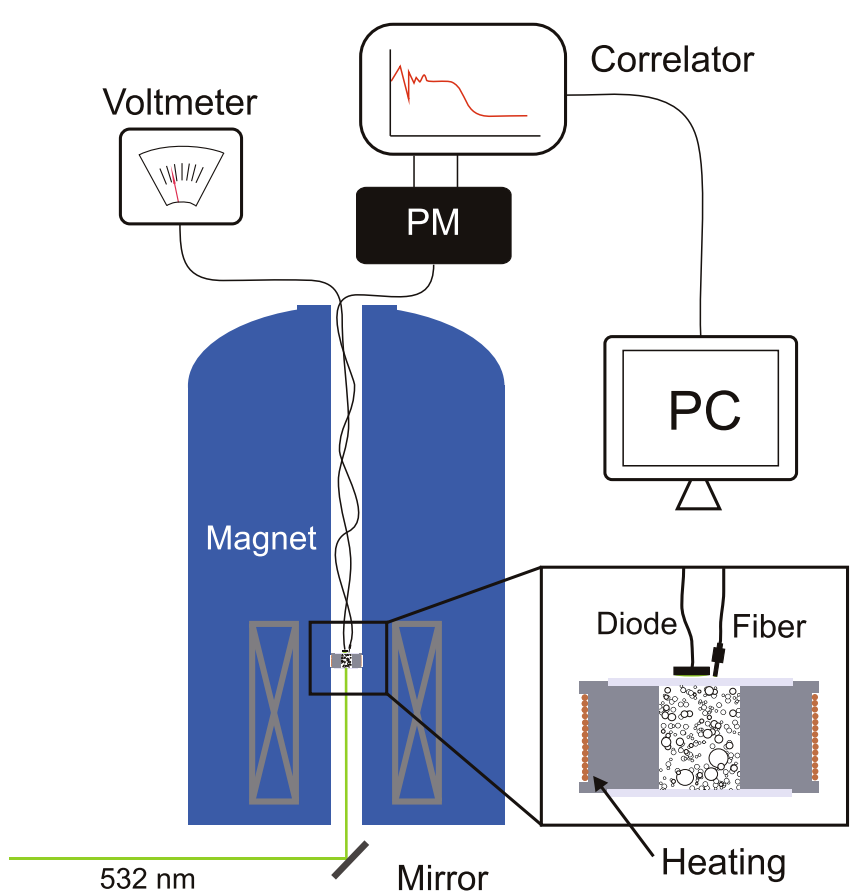

Fig. 2. Schematic illustration of the diamagnetic levitation setup. The foam sample cell, shown in detail in the inset, is illuminated from below and observed from above. The transmitted light is captured by a photodiode for the determination of the bubble size. A photomultiplier (PM) is used to determine the autocorrelation function to study the local dynamics.

capable of applying a field of $18 \mathrm{~T}$ (fig. 2). Due to the insufficient thermal insulation of the bore and the high freezing point of a water-SDS mixture, a small heating coil is added around the sample cell in order to keep the foam at constant temperature of $36(1)^{\circ}$ all times (see fig. 2). Therefore the temperature dependence of coarsening rates [32] does not influence the results below. The magnet is a superconducting solenoid at the end of which the field shows a substantial gradient [33]. This means that there is a significant upward force on the diamagnetic water-SDS mixture, given by $f=\chi B \partial B / \partial z$, where $f$ is the force density and $\chi$ is the diamagnetic susceptibility. At a specific point, when $B \partial B / \partial z=\rho g / \chi$, this force exactly compensates the gravitational force [11]. Due to the field distribution, this will lead to a stable levitation at this point [12], where the levitation will be homogeneous to one part in a thousand within a volume of $1 \mathrm{~cm}^{3}$, thus for almost the whole foam sample [33]. The residual accelerations of $0.001 \mathrm{~g}$ will still lead to macroscopic flows, however the associated inherent time scales will correspondingly be increased a thousandfold, such that the stability of the foam becomes limited by coarsening rather than drainage. Additional magnetic interaction forces between bubbles due to demagnetization effects are many orders of magnitude smaller than the force due to Laplace pressure to form or deform the bubbles and also small compared to the residual acceleration forces discussed above. Eliminating drainage this way opens up the possibility of observing the coarsening dynamics over extraordinary long times.

In order to study the time evolution of the bubble radius and the local dynamics the foam is illuminated with a Coherent Verdi solid state $\mathrm{CW}$ laser at a wavelength of $532 \mathrm{~nm}$ and a power of $100 \mathrm{~mW}$ and the transmitted light is detected either with a photodiode or a glass fiber leading to a photomultiplier and a correlator card for DWS measurements [34]. The reflective side boundaries of the container ensure that the photodiode collects the total transmitted intensity. The schematic setup is again shown in fig. 2. In addition, we directly imaged the surface bubbles for some foams using a lipstick camera, in order to characterize the absolute bubble size and polydispersity. An example is shown in fig. 1 for a foam of an initial liquid fraction of $20 \%$, at times of $10 \mathrm{~min}$ (top) and $152 \mathrm{~min}$ (middle) after creation. The time evolution of the average bubble radius obtained from such pictures is shown in fig. 1, bottom part, showing a power law increase with an exponent of $\beta=0.45(4)$. Due to the fact that our initial measurement takes place a few minutes after creation of the foam, the growth phase corresponding to the constant initial bubble radius is not observed. This is also the case for the optical measurements discussed below.

In the multiple scattering regime, the transmitted intensity is given by Ohm's law, i.e. $T=\frac{1+z e}{L / l^{*}+2 z e}[24]$, where $L$ is the thickness of the sample, $l^{*}$ is the transport mean free path and ze is a parameter describing the first scattering events and is of order unity. It was shown that $z e$ in a foam depends on liquid fraction [25], changing from 1 in the dry case to 1.5 in the liquid case. However for our experiments, the thickness $L$ is at least ten times bigger than the mean free path, such that the above expression reduces to $T \propto l^{*} / L$, independent of $z e$. Thus by determining the average transmitted intensity, we can directly obtain a measure of the change of the mean free path $l^{*}$ with foam age, since both the sample thickness and the incident intensity are fixed. The mean free path of light in the sample has been shown before to give a determination of the bubble size with $l^{*} \propto r$ [35] for the bulk of the three-dimensional foam. In fig. 3, this dependence of $l^{*}$ with foam age is shown for a set of foams with different liquid content. As can be seen in this double logarithmic plot, all foams show a scaling behavior with a power law increase of bubble size with age, $r \propto t^{\beta}$. At lower liquid fraction, the data are within errors equal to that obtained directly from the surface bubbles shown in fig. 1. For dry foams, this increase is faster with an exponent close to $\beta=1 / 2$, whereas for wet foams it is slower with $\beta=1 / 3$. These exponents are the asymptotic dynamics of the theoretical predictions for foam dynamics in the dry and wet case, respectively $[6,17-21]$. In the transition region, there is an intermediate behavior, where two distinct regimes can be seen corresponding to the two different dynamics. A possible slow cross-over between the two behaviors can be quantified by a power law fit with an intermediate exponent.

These results are summarized in fig. 4, where the exponents fitted for all experiments within a large range of liquid fractions are plotted. It can be clearly seen that 


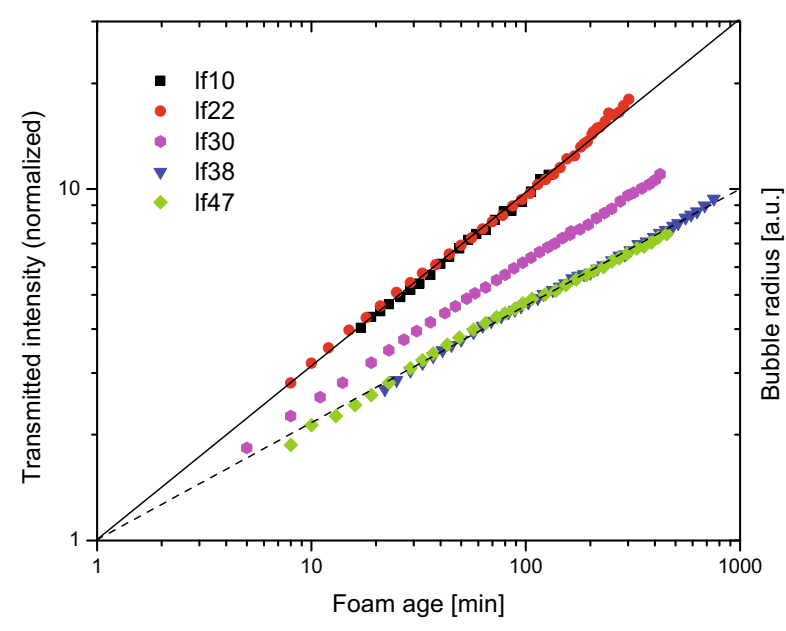

Fig. 3. The bubble size determined via the optical transmittance as a function of foam age is shown for five different liquid fractions. The plot is on doubly logarithmic scales indicating a power law dependence of the size with age. Straight lines with a slope of $1 / 2$ (solid) and $1 / 3$ (dashed) indicating the exponents of von Neumann and Ostwald dynamics are added for comparison.

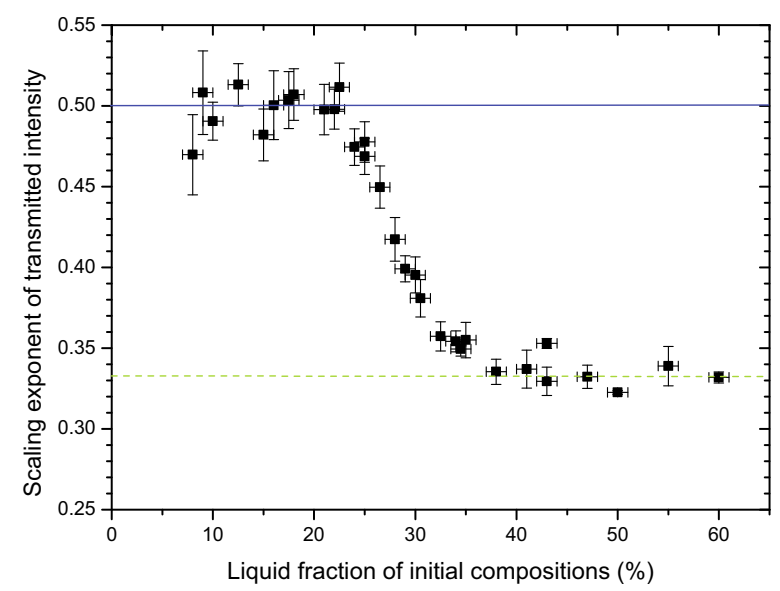

Fig. 4. The bubble growth exponent, $\beta$, determined from measurements as those shown in fig. 3 is shown as a function of liquid fraction (given in \%). For comparision, the expectation of von Neumann dynamics, $\beta=1 / 2$, and that of Ostwald ripening, $\beta=1 / 3$ is shown by the full and dashed lines, respectively. The transition between these two regimes is rather narrow at liquid fractions between $25 \%$ and $35 \%$.

below a liquid fraction of $25 \%$, the exponents are all compatible with $1 / 2$, whereas above an initial liquid fraction of $35 \%$, they are all compatible with $1 / 3$. Thus there is a clear transition in the coarsening behavior of the foams at a liquid fraction corresponding to the close packing of spheres. Both the transition and the asymptotic values of the exponents are predicted by theory [6,17-21].

Having observed this transition, it is interesting to investigate the difference in the local dynamics in the two different regimes more closely. For this purpose, we deter- mine the mean square fluctuations of the positions of the foam interfaces using DWS [30].

In DWS, the time auto-correlation function of the fluctuating transmitted intensity is determined. These fluctuations are caused by small movements of the scatterers essentially due to center-of-mass displacements of foam bubbles in the wet case and rearrangements of thin liquid walls in the dry case, respectively. Both lead to a change in the speckle interference pattern of different multiple scattering paths through the sample [29]. The DWS time auto-correlation function of the intensity in a transmission speckle spot is approximately given, in the diffusion approximation, by $g_{2}(t)=A \exp \left(-2\left\langle\delta \phi(t)^{2}\right\rangle l^{* 2} / L^{2}\right)+1$, where $\delta \phi(t)=k \Delta r(t)$ is the time-dependent phase shift incurred by the light on each scattering event on a moving particle in the different multiple scattering paths, where $k$ is the wave vector of the light used. In addition, we determine the transport mean free path as before, $l^{*} / L \propto T$, such that we obtain $\log \left(g_{2}(t)-1\right) \cdot T^{2} \propto\left\langle\Delta r^{2}(t)\right\rangle[30]$. The displacements observed correspond to a length scale of $10-100 \mathrm{~nm}$, where the uncertainty is given by the uncertainty in the determination of the absolute value of the mean free path. These fluctuations are shown for foams of different wetness (i.e. initial liquid fraction) of $22 \%, 30 \%$ and $38 \%$, in fig. 5 . The different $\left\langle\Delta r^{2}(t)\right\rangle$ shown for a single foam correspond to different ages of the foam, where each curve corresponds to a single data point in fig. 3 . The evolution of aging time is indicated by the arrow and the ages for the different foams range from 8 to $303 \mathrm{~min}$ for $22 \%$ liquid fraction, 5 to $424 \mathrm{~min}$ for $30 \%$ liquid fraction and 9 to $755 \mathrm{~min}$ for $38 \%$ liquid fraction, respectively. As can be seen, for dry foams, $\left\langle\Delta r^{2}(t)\right\rangle$ increases linearly with correlation time indicating a diffusive dynamics of the scatterers. The slope directly indicates the mobility of the scatterers, which can be seen to decrease with the age of the foam. Taking into account the increase of the bubble size with age shown above, the mobility is inversely proportional to this size. The mobility of the fluctuations is however much higher than that expected from a movement of the bubbles themselves.

For wet foams in contrast, the dynamics is rather independent of the age of the foam and the mean square fluctuations increase quadratically with time. This corresponds to a kinetic dynamics, where the scatterers travel ballistically during the observed time. Moreover, the time scale of this kinetic dynamics is essentially independent of the size of the bubbles, indicating that the bubbles move according to a small local flow present in the system. Such random ballistic motion due to intermittent rearrangements has been observed in foams of high liquid fractions before [36].

In conclusion, using diamagnetically levitated foams not affected by drainage, we have shown the existence of a transition in the bulk coarsening dynamics of threedimensional foams at an effective liquid fraction of roughly $30 \%$. At lower liquid fraction, the coarsening dynamics is governed by a von Neumann law $[6,16]$, which corresponds to a growth of the average bubble size with the square root of time. At higher liquid fraction, the bubbles grow via Ostwald ripening [17-21], i.e. the average bubble size grows with time to the power of $1 / 3$. The difference in 
a)

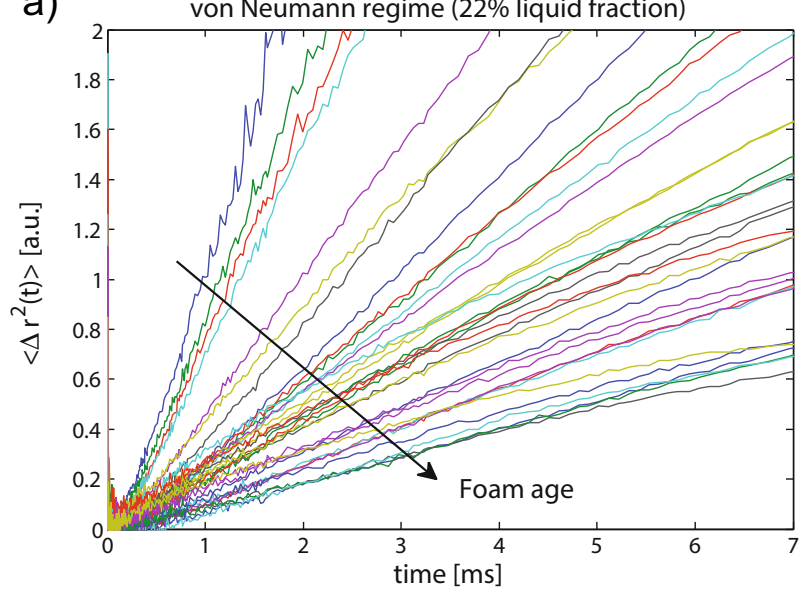

b)

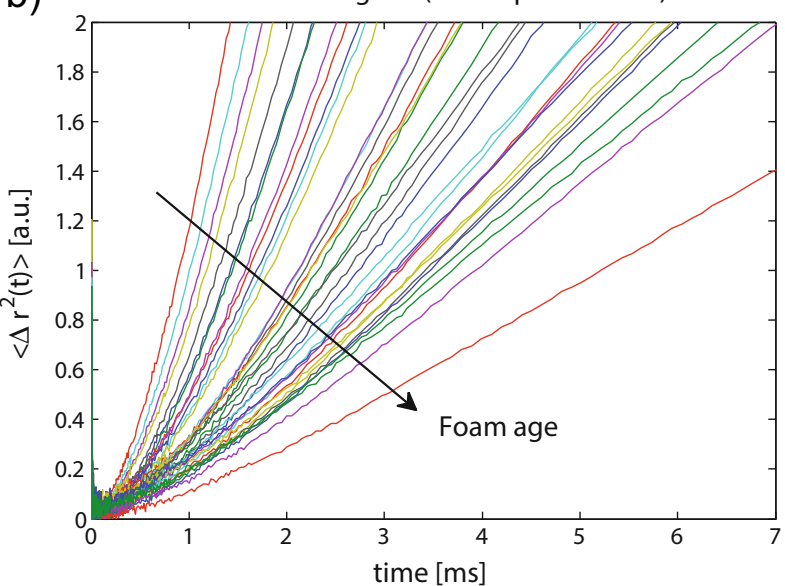

c)

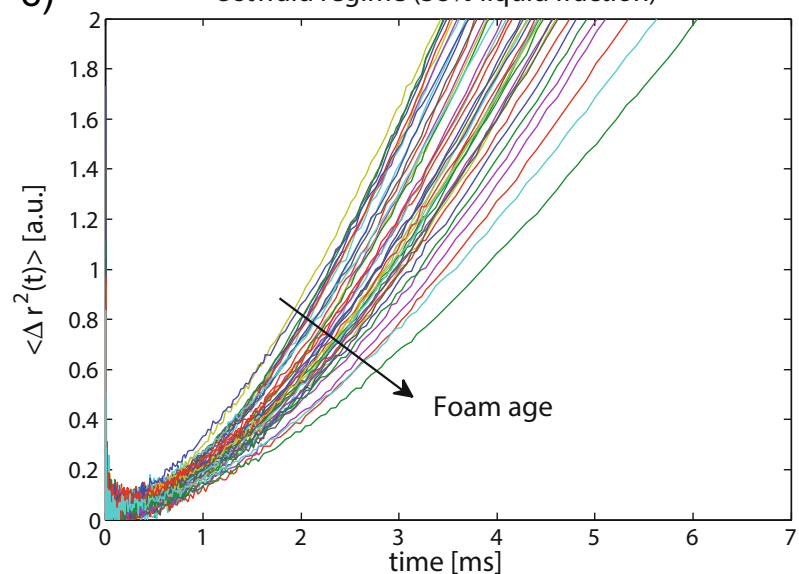

Fig. 5. The mean square displacement of scatterers in the foam is shown as a function of correlation time for three different liquid fractions (a: $22 \%$, b: $30 \%$ and c: $38 \%$ ). This shows the local dynamics of bubbles, which can be seen to be diffusive $\left(\left\langle\Delta r^{2}\right\rangle \propto t\right)$ in the von Neumann regime and ballistic $\left(\left\langle\Delta r^{2}\right\rangle \propto\right.$ $\left.t^{2}\right)$ in the Ostwald regime.

coarsening dynamics is driven by the fact that at low liquid content the bubbles are in close contact thus changing the nature of gas transport between bubbles.
In addition, we have shown that for wet foams, the local dynamics is kinetic, i.e. the bubbles essentially follow the weak convective flow of the interstitial liquid which, we think, arises from residual temperature gradients combined with slightly imperfect levitation conditions across the sample volume. The time scale of this dynamic correspondingly is independent of the age of the foam. Thus the DWS correlation time solely reflects the increase of the mean free path of light due to the increase in size.

For dry foams in contrast, where bubbles are closely packed, the dynamics of the bubbles is diffusive as would be expected in a dense out-of-equilibrium system. These movements are for instance induced by local shape fluctuations of the bubbles [32], as well as rearrangements where bubbles move to relieve accumulated stresses [37,27]. The time scale of these fluctuations also increases with the age of the foam. In the future it will be interesting to characterize the dynamics of these rearrangements, which can give a complete picture of how local dynamics influences the global dynamics of the foam. Moreover, the point of transition between wet and dry foams, i.e. the jamming point of bubbles will be interesting to investigate, however further work is needed in order to control the levitation conditions and possible magnetic interactions.

This work was funded by DFG in the context of the IRTG 667 on Soft Matter Physics of Model Systems as well as the Landesstiftung Baden-Württemberg and the Swiss National Science Foundation.

\section{References}

1. D. Weaire, S. Hutzler, The Physics of Foams (Oxford University Press, 1999).

2. S.A. Koehler et al., Phys. Rev. E 58, 2097 (1998).

3. J.A. Glazier, S.P. Gross, J. Stavans, Phys. Rev. A 36, 306 (1987).

4. A.E. Roth, C.D. Jones, D.J. Durian, Phys. Rev. E 87, 042304 (2013).

5. L.D. Landau, E.M. Lifshitz, Course in Theoretical Physics VI: Fluid Dynamics (Butterworth-Heinemann, Oxford, 2000).

6. J. von Neumann, in Metal Interfaces, edited by C. Herring, Vol. 108 (American Society for Metals, Cleveland, 1952).

7. C.P. Gonatas et al., Phys. Rev. Lett. 75, 573 (1995).

8. J. Lambert et al., Phys. Rev. Lett. 104, 248304 (2010).

9. S. Hilgenfeldt, S.A. Koehler, H.A. Stone, Phys. Rev. Lett. 86, 4704 (2001).

10. S. Hutzler, D. Weaire, Philos. Mag. Lett. 80, 419 (2000).

11. W. Braunbeck, Z. Phys. 112, 764 (1939).

12. M.V. Berry, A.K. Geim, Eur. J. Phys. 18, 307 (1997).

13. R. Straub, Diploma thesis (University Konstanz, 2008).

14. A. Saint-Jalmes, S. Marze, M. Safouane, D. Langevin, Micrograv. Sci. Technol. XVIII, 5 (2006).

15. J. Lambert et al., Phys. Rev. Lett. 99, 058304 (2007).

16. R.D. MacPhearson, D.J. Srolovitz, Nature 446, 1053 (2007).

17. W. Ostwald, Z. Phys. Chem. 37, 385 (1901).

18. I.M. Lifshitz, V.V. Slyozov, J. Phys. Chem. Solids 19, 35 (1961). 
19. C. Wagner, Z. Elektr, Inf.-Energietech. 65, 581 (1961).

20. A.J. Markworth, Metallography 3, 197 (1970).

21. W.W. Mullins, J. Appl. Phys. 59, 1341 (1986).

22. A. Knaebel et al., Europhys. Lett. 52, 73 (2000).

23. I. Fortuna et al., Phys. Rev. Lett. 108, 248301 (2012).

24. P. Sheng, Introduction to Wave Scattering, Localization and Mesoscopic Phenomena (Academic Press, 1995).

25. M.U. Vera, A. Saint-Jalmes, D.J. Durian, Appl. Opt. 40, 4210 (2001).

26. D.J. Durian, D.A. Weitz, D.J. Pine, Phys. Rev. A 44, R7902 (1991).

27. S. Cohen-Addad, R. Höhler, Phys. Rev. Lett. 86, 4700 (2001).

28. A. Stucco et al., Soft Matter 7, 631 (2011).

29. G. Maret, P.E. Wolf, Z. Phys. B 65, 409 (1987).
30. D.J. Pine, D.A. Weitz, J.X. Zhu, E. Herbolzheimer, J. Phys. 51, 2101 (1990).

31. A. Saint-Jalmes, M.U. Vera, D.J. Durian, Eur. Phys. J. B 12, 67 (1999)

32. A.D. Gopal, D.J. Durian, J. Opt. Soc. Am. A 14, 150 (1997).

33. C.C. Maass, N. Isert. G. Maret, C.M. Aegerter, Phys. Rev. Lett. 100, 248001 (2008).

34. G. Maret, Curr. Op. Colloid Int. Sci 2, 251 (1997).

35. A.S. Gittings, R. Bandyopadhyay, D.J. Durian, Europhys. Lett. 65, 414 (2004).

36. M. Le Merrer, S. Cohen-Addad, R. Höhler, Phys. Rev. Lett. 108, 188301 (2012)

37. D.J. Durian, D.A. Weitz, D.J. Pine, Science 252, 686 (1991). 